\title{
The Modification of Special Relativity
}

\author{
Jian'an Wang \\ Department of Physics, Shenzhen University, Shenzhen, China \\ Email: wja@szu.edu.cn
}

How to cite this paper: Wang, J.A. (2019) The Modification of Special Relativity. Journal of Modern Physics, 10, 1615-1644. https://doi.org/10.4236/jmp.2019.1014107

Received: October 8, 2019

Accepted: November 26, 2019

Published: November 29, 2019

Copyright $\odot 2019$ by author(s) and Scientific Research Publishing Inc. This work is licensed under the Creative Commons Attribution International License (CC BY 4.0).

http://creativecommons.org/licenses/by/4.0/

\begin{abstract}
In this paper, the phenomenon of light velocity invariance is analyzed by using a new etheric view, and the physical mechanism of light velocity invariance is given, the principle of constancy of light velocity and the principle of special relativity are modified, and the Lorentz transformation is deduced by using these two modified principles. The relationship between mass and speed, the relationship between time and speed, and the relationship between length and speed are reanalyzed, and physical explanations different from the Special Relativity are given. Experiments for verification of the new theory are designed and presented. Experiment 1: Verify that the kinetic energy of a particle is only related to the speed of the particle relative to the ether, independent of the observer's motion. Experiment 2: Verify that magnetic field is produced by electric charge's motion relative to ether, and is independent of the observer. Experiment 3: Redoing the Michelson-Morley experiment on the space station to prove the existence of the ether. Experiment 4: Simulation of the generation and reversal of the Earth's magnetic field, thus proving that the magnetic field is generated by the relative motion of the electric charge to the ether. Experiment 5: Simulate the formation of the natural iron meteorites and show that the atom becomes smaller with the increase of the etheric density of the space. Experiment 6: Aberrations contrast experiment to prove that for the observers on Earth, according to the theory of this paper, stars have aberration but the light sources on Earth have no aberration; according to the theory of special relativity, there are aberrations for both the stars and the light sources on Earth.
\end{abstract}

\section{Keywords}

Principle of Constancy of Light Velocity, Principle of Special Relativity, Special Relativity, Lorentz Transformation

\section{Introduction}

In the 19th century, with the development of the wave theory of light, "ether" 
theory [1] became popular again. At that time, many physicists believed that there must exist an elastic material "ether" in space that could propagate light waves, and regarded "ether" as the absolutely static inertial system. To verify the existence of "ether" by experiments became the goal pursued by many scientists at that time. Since the Earth orbits the sun at a speed of 30 kilometers per second, it was thought that the Earth must encounter an "etheric wind" of 30 kilometers per second. In 1887 Michelson and Morley did the famous Michelson-Morley experiment [2] to measure the ether wind on Earth, but it turned out that ether wind does not exist and that the speed of light is the same in different directions on the surface of the Earth. In this historical context, Einstein abandoned the ether absolute stationary frame of reference and established the special relativity. In 1905 Einstein published the article "On Electrodynamics of Moving Bodies" [3] which is called "special relativity" by later generations. The starting point of this theory is two basic assumptions: the principle of special relativity and the principle of constancy of light velocity. The core equations of the theory are the Lorentz transformations [4]:

$$
\left\{\begin{array}{l}
x=\frac{x^{\prime}+v t}{\sqrt{1-(v / c)^{2}}} \\
y=y^{\prime} \\
z=z^{\prime} \\
t=\frac{t^{\prime}+v x^{\prime} / c^{2}}{\sqrt{1-(v / c)^{2}}}
\end{array}\right.
$$

Special relativity predicted some new effects (relativistic effects) that Newton classical physics did not have, such as time dilation, length contraction, mass speed relation, mass energy relation and so on. Those relativistic effects have been verified by many relevant experiments, for example, the increase of the lifetime of the flying muon proves the correctness of the time dilation relativistic effect, the increase of the mass of the accelerated particles on the accelerator with the increase of speed proves the correctness of the relativistic effect of the relationship between mass and speed, and the explosion of atomic bomb proves the correctness of the relationship of special relativity between mass and energy. Today, special relativity is one of the foundations of modern physical theory.

The author believes that there is no end to science, and the spirit of science is to keep exploring. Although special relativity has achieved great success, it is by no means the end of physics. The core of special relativity is the principle of constancy of light velocity. The author believes that there should be a deep natural mystery behind the principle of constancy of light velocity, but in special relativity, the physical mechanism of this principle is not given. In addition, the principle of constancy of light velocity of special relativity is contradictory to people's daily experience and logic, and is incomprehensible. Besides, special relativity has no further physical explanations of its main conclusions, such as time dilation, length contraction and mass speed relation. In order to get the physical 
mechanism of the principle of constancy of light velocity and discover the mystery behind it, the author started this research with great curiosity.

In this research, the first step needed is to get the physical mechanism of light velocity invariance so as to modify the principle of constancy of light velocity and the principle of special relativity. The second step needed is to derive the Lorentz transforms from the modified principle of constancy of light velocity and the modified principle of special relativity. The third step needed is to reinterpret the time dilation, the length contraction, the mass speed relation with the new Lorentz transforms. The final step is to design and present related experiments to verify the new theory.

\section{On the Principle of Constancy of Light Velocity}

\subsection{The Physical Mechanism of Constancy of Light Velocity}

The principle of constancy of light velocity can be expressed as "In any inertial frame, the propagation speed of light in vacuum is constant and does not change with the relative motion of the light source and the observer".

How do we understand this incomprehensible principle? The author holds that dialectical materialism is correct and that the world is made up of matter (including energy). One of the purposes of physical research is to understand the physical mechanism behind any hypothesis or mathematical formula of physics. The author believes that space and time are the properties of matter (including energy) and are not independent existence. Therefore, the frame of reference in physics must be made up of matter (including energy) rather than a purely mathematical concept.

Aristotle (384-322 BC), an ancient Greek philosopher of great knowledge, believed that vacuum does not exist at all, and that space is filled with continuous matter (ether) everywhere [1]. In the fourth century BC, two Chinese Song Yan and Yin Wen put forward the theory of "ultimate gas" believing that [1] the celestial bodies and all things in the world are composed of a kind of so-called "ultimate gas" substance. When the "ultimate gas" gathers it turns into tangible objects, and when the objects dissipate, they become "ultimate gas" and return to space. According to the mass and energy relation formula of special relativity [3], mass and energy can be converted into each other, so we can assume that the "ultimate gas" referred to by the two ancient Chinese philosophers is energy (ether).

From the energy conservation equation of Compton Scattering [5]:

$$
\frac{h c}{\lambda}+m_{0} c^{2}=\frac{h c}{\lambda^{\prime}}+\frac{m_{0} c^{2}}{\sqrt{1-(v / c)^{2}}}
$$

in the formula, $\lambda$ is the wavelength of incident light wave, $m_{0}$ is the static mass of electron, $c$ is the speed of light in vacum, $v$ is the speed of the electron relative to the laboratory (Earth), $\lambda^{\prime}$ is the wavelength of outgoing light wave, and $h$ is the Planck parameter. From the above formula, we can see that in 
Compton Scattering experiment, when the photon collide with the electron, the total energy of the system (the photon and the electron) is calculated by using the electron as the frame of reference, that is to say, the electron is always regarded as static, and the speed of the photon relative to the electron is always at $\mathrm{c}$ (the speed of light in vacum) regardless of the relative motion between the photon and the electron. But after the collision when the photon and the electron are separated, the total energy of the system (the photon and the electron) is calculated by using the laboratory (Earth) as the frame of reference, that is to say, the velocity of the electron is calculated by using the laboratory (Earth) as the frame of reference and the speed of the photon relative to the laboratory (Earth) is always at $c$ (the speed of light in vacum). From the Compton Scattering experiment, we can guess that the electron has an etheric layer that moves with it, and that the Earth also has an etheric layer that moves with it.

To sum up, we can assume that:

1) The cosmic space is full of ether (background energy), and ether is also the medium in which light waves travel.

2) All fields (gravitational field, electromagnetic field, strong interaction field and weak interaction field etc.) contain and compress ether (energy).

3) Each particle or object carries an etheric layer (sphere of influence) that surrounds and moves with it.

The following is a physical explanation of the principle of constancy of light velocity based on the above new etheric view: Because ether is full of space and the speed of the light wave relative to the ether medium is constant, so the speed of the light wave relative to the ether medium is independent of the motion of the light source. And because all fields contain and compress ether, any object or particle will attract and drag an etheric layer that surrounds it to move together. The thickness of the etheric layer dragged together is related to the total energy of the particle or object. In the etheric layer of an object or particle, the object or particle has the greatest influence, and the influence of other objects or particles can be ignored. For example, within the range of hundreds of kilometers above the surface of the Earth, the Earth (gravitational field) has the greatest influence, and the influence of the sun (gravitational field) and other planets (gravitational field) is negligible. Therefore, to study the motion of objects (such as cars, aircraft, satellites) in this range can be based on the etheric layer of the Earth as the absolute frame of reference. Usually the thickness of the etheric layer is of the same order of magnitude as the scale of the object. So when a photon approaches a particle or object, it will enter the etheric layer of the particle or object and adjust its speed to maintain the relative speed to the newly entered etheric layer at the constant speed of light c. So, no matter what velocity a particle or object moves at, the speed at which a photon moves relative to it when colliding with it or approaching it is constant at the constant speed of light $\mathrm{c}$. This is the mystery of the constancy of light velocity.

In the Michelson-Morley experiment [2] the etheric wind was not detected because the Earth carries its own etheric layer with it, and the ether of the Earth 
is almost completely stationary relative to the Earth's surface (the influence of the sun's ether is negligible on the Earth's surface). So light travels at the same speed relative to the Earth in any direction along the Earth's surface.

We can use the following case to better understand the physical mechanism of the principle of constancy of light velocity. Suppose a submarine moves together with a container filled with water, and a sound speed detector is installed in the container, then no matter how the submarine moves and no matter how the underwater sound source (like dolphin or whale) moves, the sound speed measured by the detector is constant. If we take the water as the ether, the sea as the universe, the container as the object, then we can very easily understand the principle of constancy of light velocity.

It can be seen that the constant velocity of light is actually a physical phenomenon in the universe, which is produced by the photon together with the object and the ether. The physical phenomenon of constant velocity of light can also be regarded as a proof of the existence of the ether and a proof that every particle or object drags a layer of ether to move together. The so-called constant velocity of light is actually that the photon's velocity relative to the ether remains constant, but its velocity relative to any fixed inertial system is variable. In a selected inertial system, the velocity of light varies as photons move from etheric system A to etheric system B, which is moving relative to etheric system A. For example, when we choose the solar etheric system as the frame of reference, then when a photon emits from the sun, it first moves at the speed of $c$ in the etheric system of the sun, and then it goes into the etheric system of the Earth and moves at the speed of $c$ in the etheric system of the Earth. Because the Earth has relative motion to the sun, the speed of the photon relative to the solar etheric system changes when the photon enters the etheric system of the Earth from the solar etheric system. So the physical phenomenon that the velocity of light is constant does not mean that the velocity of light relative to any inertia system remains constant in a vacuum, but that photons adjust their velocity when moving into the etheric layer of any object or particle so that the velocity relative to the etheric layer of the object or particle is always at the constant velocity of light $c$. In other words, any measurement of the collision velocity between a photon and an object will change the velocity of light so that the velocity of the photon relative to the etheric layer of the object is always adjusted to $c$ the constant velocity of light, when the photon approaches the object and enters into the erthric layer of the object. Therefore, the principle of constancy of light velocity in special relativity is incorrect and needs to be corrected.

\subsection{The Modification of the Principle of Constancy of Light Velocity}

If we define the etheric layers that are dragged by different particles or objects and are moving with the particles or objects the different etheric systems, such as the etheric system of electron, the etheric system of nucleus, the etheric system of crystals, the etheric system of Earth, the etheric system of the sun, the etheric 
system of galaxies, and define the space of the universe the etheric system of the universe, then "the propagation velocity of light in all etheric systems remains constant $C(299,792,458 \mathrm{~m} / \mathrm{s})$, which has nothing to do with the motion of the light source and the motion of the etheric system" this is the modified principle of constancy of light velocity or called "principle of constancy of light velocity in ether".

\section{On the Principle of Special Relativity}

\subsection{The Problem Exists in the Principle of Special Relativity}

The principle of special relativity can be expressed as follows: the law of physics has the same form in any inertia system.

Here is one equation of the Maxwell equations in a vacuum [6]

$$
\nabla \times \boldsymbol{B}=\mu_{0} \boldsymbol{J}+\varepsilon_{0} \mu_{0} \frac{\partial \boldsymbol{E}}{\partial t}
$$

In the above equation, $\boldsymbol{B}$ is the magnetic induction, $\boldsymbol{J}$ is the current density, $\varepsilon_{0}$ is the dielectric constant of the vacuum, $\mu_{0}$ is the magnetic permeability of the vacuum, $\boldsymbol{E}$ is the electric field strength, and $t$ is time. Since [7]

$$
c=\frac{1}{\sqrt{\varepsilon_{0} \mu_{0}}}
$$

In the above formula $\mathrm{c}$ is the speed of light in vacuum. If the speed of light in vacuum cannot be maintained the same relative to different inertial frame of references, that is to say if the principle of constancy of light velocity is not true, then the mathematical form of the above Maxwell equation cannot be maintained the same in all inertial systems, in other words, the principle of special relativity is incorrect.

The status of various etheric systems (for example the etheric system of the Earth and etheric system of the Milky Way) is unequal due to the great differences in the quantity of energy contained in the etheric systems. For example, we can study the motion of the Earth with the sun as a reference frame, but we cannot study the motion of the sun with the Earth as a reference frame. We can study the motion of a car with the Earth as a frame of reference, but we can never study the motion of the Earth with the car as a frame of reference. Therefore, the inertia systems of equal status in special relativity do not exist in the real physical world.

In addition, the principle of special relativity is not true in the real physical world because it ignores the existence and influence of spatial background energy (ether), such as the influence of ether on the time, size and inertia mass of objects that move in the ether, as well as the influence of magnetic field, rotating electric field and electromagnetic wave on the charged body moving relative to it.

From the point of view of the new etheric view of this paper, the prolongation of time, the contraction of length and the increase of the inertia mass of a mov- 
ing object are not the relative observational effects described in the special relativity, but the etheric effect, which is the absolute physical effect produced by the ether on the object when the object moves relative to the ether, and is independent of the motion state of the observer.

In summary, the principle of special relativity is incorrect and needs to be modified.

\subsection{The Modification of the Principle of Special Relativity}

If we define the etheric layers that are dragged by different particles or objects and are moving with the particles or objects the different etheric systems, such as the etheric system of electron, the etheric system of nucleus, the etheric system of crystals, the etheric system of Earth, the etheric system of the sun, the etheric system of galaxies; and define the space of the universe the etheric system of the universe, then the Maxwell equations can keep their mathematical forms unchanged in different etheric systems. Therefore, we can draw the conclusion that the form of any law of physics is the same in all kinds of etheric systems, which is the modified principle of special relativity, or called "the principle of relativity of etheric systems".

\subsection{The Correct Selection of the Etheric System}

Because the spatial scales of different etheric systems are very different (from the microcosm to the universe), the amount of energy (ether) contained in different etheric systems is also very different, different etheric systems are not equal. For example, it is impossible to equalize the etheric system of the Earth and the etheric system a car, because the Earth cannot move in the etheric system of the car, the physical laws of the Earth moving around the sun cannot exist in the etheric system of the car, but can exist in the etheric system of the sun. Therefore, when dealing with the kinematic dynamics of an automobile that moves relative to the Earth, we can only take the Earth etheric system as the reference frame, not the automobile etheric system as the reference frame, otherwise there will be problem of energy conservation.

All the etheric systems are the absolute frame of references, that are the working frameworks for scientific workers. It is very important to choose the proper etheric system when doing scientific research.

The physical world is very similar to human society in some aspects. Human society maintains order through laws, and the physical world maintains order through the laws of physics. For human society, the greater the power a government has the greater the influence. For physical world the more the energy (ether) a system has the greater the influence. The human society of a country is controlled by the national government, the state government, the city government, the township government and the village government etc. The universe is controlled by the cosmic etheric system, the galaxy group etheric system, the galaxy etheric system, the star etheric system, the planetary etheric system, the 
crystal etheric system and the nuclear etheric system etc. Ether in the universe is similar as power in human society, the range and density of the ether distribution in universe is equal to the range of power and the influence in human society. For the human society, a normal individual can not affect the national machine's operation. For the universe, a car on the Earth can not affect the Earth's movement. For human society, the villagers are directly controlled by the village chief rather than the president of the country, and the village chief has far greater control over the villagers than the president. In the physical world, the electrons in the atom are directly bound by the nucleus (nuclear electrostatic field and ether contained in the electrostatic field), not the universe or the sun or the Earth. The influence of the nucleus to the electrons is far greater than the universe or the sun or the Earth. It can be seen that the principle of special relativity that all inertial systems are equal is not true, and the principle of general relativity that all non-inertial systems are equal is also untrue. Therefore, we must follow the following rules when choosing a frame of reference:

When we study the motion of galaxy clusters in the universe (or the motion of isolated objects far away from all galaxies), we must adopt the etheric system of the universe as the absolute reference system.

When we study the motion of galaxies in the galaxy cluster, we must adopt the etheric system of the galaxy cluster as the absolute reference system.

When we study the motion of stars in the galaxy, we must adopt the etheric system of the galaxy as the absolute reference system.

When we study the motion of the planets in the solar system, we must adopt the etheric system of the sun as the absolute reference system.

When we study the motion of the satellites around the planet, we must adopt the etheric system of the planet as the absolute reference system.

When we study the motion of the macroscopic objects on or near the Earth's surface, we must adopt the etheric system of the Earth as the absolute reference system.

When we study the motion of the free microscopic particles (such as cosmic rays entering the Earth's atmosphere) on or near the Earth's surface, we must adopt the etheric system of the Earth as the absolute reference system also.

When we study the motion of the particles accelerated by an cyclotron, we must adopt the etheric system formed by the magnetic field of the cyclotron as the absolute reference system.

When we study the motion of atoms or electrons in a crystal, we must adopt the etheric system formed by the crystal as the absolute reference system. For example when we study the motion of electrons in the wire, we must use the etheric system formed by the wire as the absolute reference system.

When we study the motion of the electrons bound by a nucleus, we must adopt the etheric system of the nucleus as the absolute reference system.

Therefore, in the Compton Scattering experiment [5], when the photon and the electron collide, the photon enters the etheric layer of the electron, the speed 
and the energy of the electron and the photon must be calculated with the etheric layer of the electron as the absolute frame of reference. But, after the collision when the photon and electron are separated from each other, they enter the etheric layer of the Earth, respectively. At this time, the speed and energy of the electron and the photon must be calculated by using the etheric layer of the Earth as the absolute frame of reference.

For the human society, the residents of the fuzzy zone located on both sides of the border are often affected and bound by the two places. For the physical world the influence of both etheric systems must be taken into account in a space where the influence of both etheric systems cannot be ignored. For example, when studying the propagation of light in water flow of water pipes, the combined effects of Earth ether and water ether must be considered.

Because of the influence of background etheric system, the speed between the etheric layer dragged by rotating object and the surface of the rotating object will increase with the increase of the distance between etheric layer and the surface of the object, so it is necessary to make appropriate correction when choosing an etheric system for calculation. For example, when studying the motion of artificial satellites, it is necessary to take into account the influence of the fact that the farther away the satellite is from the Earth, the higher the speed of the etheric layer relative to the Earth, and make appropriate modifications to the calculation results.

In addition, among all the above etheric systems, only the cosmic etheric system is an isolated system, so all the conservation laws (such as energy conservation, momentum conservation, and angular momentum conservation, etc.) are strictly valid only in the cosmic etheric system.

\section{On the Lorentz Transformation}

\subsection{The Derivation of Lorentz Transformation from the Modified Principle of Special Relativity and the Modified Principle of Constancy of Light Velocity}

The lorentz coordinates and time transformations can be derived from the modified principle of special relativity and the modified principle of constancy of light velocity.

\subsubsection{The Transformation Relationship between Space-Time Coordinates}

If we assume that both time and space are uniform in a small etheric space, as shown in Figure 1, the transformation between space-time coordinates can be approximately linear. Let $S$ and $S$ ' be two etheric systems, one large and one small, the small etheric system $S^{\prime}$ (for example, the Earth etheric system) is in the large etheric system $S$ (for example, the solar etheric system) and is moving in a straight line with uniform speed v relative to $S$. For any event, the space-time coordinates of $P$ in $S$ system and $S^{\prime}$ system are $(x, y, z, t)$ and $\left(x^{\prime}, y^{\prime}, z^{\prime}, t^{\prime}\right)$ respectively. Since $S^{\prime}$ is moving along the $x$-axis with constant speed relative to $S$, 


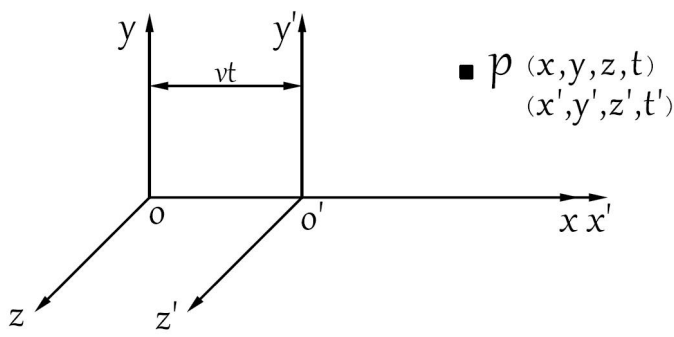

Figure 1. Transformation of space-time coordinates.

obviously $y^{\prime}=y, \quad z^{\prime}=z$. The coordinates of the origin of $S$ system observed in $S$ system are: $x=0$; and observed in $S^{\prime}$ system are: $x^{\prime}=-v t^{\prime}$ or $x^{\prime}+v t^{\prime}=0$. So we have $x=x^{\prime}+v t^{\prime}$ 。For any point in the space, we can set:

$$
x=k\left(x^{\prime}+v t^{\prime}\right)
$$

In the above formula, $k$ is a constant of proportionality. Similarly, we can get:

$$
x^{\prime}=k^{\prime}(x-v t)=k^{\prime}(x+(-v) t)
$$

According to the "modified principle of special relativity" any law of physics in $S$ system and $S$ 'system should be the same in the form, so the form of (4.1) and (4.2) should be the same (except positive and negative signs), so $k=k^{\prime}$.

\subsubsection{To Obtain the Constant $k$ from the Modified Principle of Constancy of Light Velocity}

Let $S$ and $S^{\prime}$ be two etheric systems, one large and one small, the small etheric system $S$ '(for example, the Earth etheric system) is in the large etheric system $S$ (for example, the solar etheric system) and is moving in a straight line with uniform speed relative to $S$. Suppose a light signal travels along the $x$ 'axis from the origin in the $S$ 'system, then at any instant $t$, the coordinate of the point the signal reaches in the $S^{\prime}$ system is: $x^{\prime}=c t^{\prime}$, where $c$ is the speed of light in ether (vacum). Suppose another light signal travels along the $x$ axis from the origin in the $S$ system, then at any instant $t$, the coordinate of the point the signal reaches in the $S$ system is: $x=c t$, where $c$ is the speed of light in ether (vacum). Suppose that at the instant in which the origin of the $S$ system and the origin of $S$, system meet, one light signal travels along the $x^{\prime}(x)$ axis from the origin. Suppose that the $S$ system disappear suddenly when the light signal start to travel from the origin, so the light travels in $S^{\prime}$ system, and we have $x^{\prime}=c t^{\prime}$. Suppose that $S$ system is there but the $S$ 'system disappear suddenly when the light signal start to travel from the origin, so the light travels in $S$ system, and we have $x=c t$. So we have:

$$
x x^{\prime}=c^{2} t t^{\prime}=k^{2}(x-v t)\left(x^{\prime}+v t^{\prime}\right)=k^{2}(c t-v t)\left(c t^{\prime}+v t^{\prime}\right)=k^{2} t t^{\prime}\left(c^{2}-v^{2}\right)
$$

From above equation we can get:

$$
k=\frac{c}{\sqrt{c^{2}-v^{2}}}=\frac{1}{\sqrt{1-(v / c)^{2}}}
$$

and then, we can get: 


$$
\begin{gathered}
x^{\prime}=\frac{x-v t}{\sqrt{1-(v / c)^{2}}} \\
x=\frac{x^{\prime}+v t^{\prime}}{\sqrt{1-(v / c)^{2}}}
\end{gathered}
$$

By eliminating $x^{\prime}$ from the above two equations, we get:

$$
t^{\prime}=\frac{t-v x / c^{2}}{\sqrt{1-(v / c)^{2}}}
$$

By eliminating $x$, then we get:

$$
t=\frac{t^{\prime}+v x^{\prime} / c^{2}}{\sqrt{1-(v / c)^{2}}}
$$

Based on the above results, the Lorentz transformations are obtained:

$$
\left\{\begin{array}{l}
x^{\prime}=\frac{x-v t}{\sqrt{1-(v / c)^{2}}} \\
y^{\prime}=y \\
z^{\prime}=z \\
t^{\prime}=\frac{t-v x / c^{2}}{\sqrt{1-(v / c)^{2}}}
\end{array}\right.
$$

and the Lorentz inverse transformations are obtained:

$$
\left\{\begin{array}{l}
x=\frac{x^{\prime}+v t^{\prime}}{\sqrt{1-(v / c)^{2}}} \\
y=y^{\prime} \\
z=z^{\prime} \\
t=\frac{t^{\prime}+v x^{\prime} / c^{2}}{\sqrt{1-(v / c)^{2}}}
\end{array}\right.
$$

It can be seen that the Lorentz transformations are the direct result of the modified principle of special relativity (i.e. the form of any law of physics is the same in all kinds of etheric systems) and the modified principle of constancy of light velocity (i.e. the propagation velocity of light in all etheric systems remains constant $C(299,792,458 \mathrm{~m} / \mathrm{s})$, which has nothing to do with the motion of the light source and the motion of the etheric system).

\subsection{Lorentz Velocity Transformations}

\subsubsection{The Derivation of Lorentz Velocity Transformations}

Lorentz transformation is the relationship of the space-time coordinates of events between different etheric systems, and according to Lorentz coordinates transformations and Lorentz time transformation, Lorentz velocity transformations can be obtained. Let the velocity of an object in $S$ system and $S^{\text {' }}$ system be $\left(u_{x}, u_{y}, u_{z}\right)$ and $\left(u_{x}^{\prime}, u_{y}^{\prime}, u_{z}^{\prime}\right)$ respectively, according to Lorentz coordinates 
transformations and time transformation, we can get:

$$
\begin{gathered}
\mathrm{d} x^{\prime}=\frac{\mathrm{d} x-v \mathrm{~d} t}{\sqrt{1-(v / c)^{2}}}=\frac{(\mathrm{d} x / \mathrm{d} t-v) \mathrm{d} t}{\sqrt{1-(v / c)^{2}}}=\frac{\left(u_{x}-v\right) \mathrm{d} t}{\sqrt{1-(v / c)^{2}}} \\
\mathrm{~d} t^{\prime}=\frac{\mathrm{d} t-v \mathrm{~d} x / c^{2}}{\sqrt{1-(v / c)^{2}}}=\frac{\mathrm{d} t\left(1-v u_{x} / c^{2}\right)}{\sqrt{1-(v / c)^{2}}}
\end{gathered}
$$

so:

$$
\frac{\mathrm{d} x^{\prime}}{\mathrm{d} t^{\prime}}=\frac{\left(u_{x}-v\right) \mathrm{d} t}{\sqrt{1-(v / c)^{2}}} / \frac{\mathrm{d} t\left(1-v u_{x} / c^{2}\right)}{\sqrt{1-(v / c)^{2}}}
$$

that is:

$$
u_{x}^{\prime}=\frac{u_{x}-v}{1-v u_{x} / c^{2}}
$$

$y^{\prime}=y, \quad z^{\prime}=z$, so $\mathrm{d} y^{\prime}=\mathrm{d} y, \mathrm{~d} z^{\prime}=\mathrm{d} z$, then:

$$
\frac{\mathrm{d} y^{\prime}}{\mathrm{d} t^{\prime}}=\mathrm{d} y / \frac{\mathrm{d} t\left(1-v u_{x} / c^{2}\right)}{\sqrt{1-(v / c)^{2}}}
$$

that is:

$$
u_{y}^{\prime}=\frac{u_{y} \sqrt{1-(v / c)^{2}}}{1-v u_{x} / c^{2}}
$$

In the same way we can get:

$$
u_{z}^{\prime}=\frac{u_{z} \sqrt{1-(v / c)^{2}}}{1-v u_{x} / c^{2}}
$$

Therefore, we get the Lorentz velocity transformations:

$$
\left\{\begin{array}{l}
u_{x}^{\prime}=\frac{u_{x}-v}{1-v u_{x} / c^{2}} \\
u_{y}^{\prime}=\frac{u_{y} \sqrt{1-(v / c)^{2}}}{1-v u_{x} / c^{2}} \\
u_{z}^{\prime}=\frac{u_{z} \sqrt{1-(v / c)^{2}}}{1-v u_{x} / c^{2}}
\end{array}\right.
$$

and the inverse transformations:

$$
\left\{\begin{array}{l}
u_{x}=\frac{u_{x}^{\prime}+v}{1+v u_{x}^{\prime} / c^{2}} \\
u_{y}=\frac{u_{y}^{\prime} \sqrt{1-(v / c)^{2}}}{1+v u_{x}^{\prime} / c^{2}} \\
u_{z}=\frac{u_{z} \sqrt{1-(v / c)^{2}}}{1+v u_{x}^{\prime} / c^{2}}
\end{array}\right.
$$




\subsubsection{Discussions}

1) When the velocities $u$ and $v$ are far less than $c$ (the velocity of light), Lorentz velocity transformation (4.14) is transformed into Galileo velocity transformation:

$$
u_{x}^{\prime}=u_{x}-v
$$

2) Using the Lorentz velocity transformation (4.15), it can be proved that the speed of light is $c$ in any etheric system. Suppose that the speed of a light signal propagated along the $x^{\prime}$ 'direction measured by the observer in the $S^{\prime}$ system is $c$, and the speed of the light signal measured by the observer in the $S$ system is:

$$
u_{x}=\frac{c+v}{1+v c / c^{2}}=c
$$

That is to say, the speed of light in $S$ system and $S$ 'system is the same.

\subsection{The Physical Meaning and Proper Use of Lorentz Transformations}

From the point of view of the new etheric view of this paper, the etheric system is unequal with any inertial or non-inertial system. Because any object that is moving in ether will produce associated physical effects (such as the changes in time, length, and inertial mass). Because only objects moving in ether can produce related physical effects, only the motion of objects in ether is physically meaningful. As an object moves from one etheric system to another etheric system that moves relative to the primary etheric system, the speed of the object relative to ether changes, The change of the speed of the object relative to ether will result in the change of the energy (etheric) density of the local space in which the object is located, resulting in the change of the local space and time. Therefore, the Lorentz transformations must be applied between the etheric system and any other etheric system that is moving uniformly in a straight line with respect to it (since there is no object in the universe that is really moving uniformly in a straight line, it can only be approximated). For example that a photon from a distant star outside the Milky Way galaxy reaches the Earth. The photon must first leave the etheric system of the star to enter the etheric system of the galaxy in which the star is located, and then enter the cosmic etheric system from the etheric system of the galaxy in which the star is located. And then from the cosmic etheric system to enter the Milky Way galaxy etheric system, from the Milky Way galaxy etheric system to enter the solar etheric system, and from the solar etheric system to enter the Earth etheric system. If we want to calculate exactly the velocity of the photon relative to the Earth to get a precise Aberration Angle, the Lorentz velocity transformation must be used to calculate the velocity of the photon to enter galaxy etheric system from the star etheric system, then to calculate the velocity of the photon to enter the cosmic etheric system from the galaxy etheric system, then to calculate the velocity of the photon to enter the Milky Way galaxy etheric system from the comic etheric system, 
and then to calculate the velocity of the photon to enter the solar etheric system from Milky Way galaxy etheric system, and finally to calculate the velocity of the photon to enter the Earth etheric system from the solar etheric system. We cannot directly calculate the velocity of the photon relative to the Earth's etheric system by the lorentz transformation between the etheric system of the star that emits the photon and the Earth's etheric system. So Lorentz transformations are not appropriate for two etheric systems that are not directly related, nor is Lorentz transform suitable for any other two inertial systems that are not etheric systems. The transformation between any other two inertial systems that are not the etheric system (without considering the influence of the ether) is only suitable for the Galilean transformation. For example, assuming that the velocityies of particles A and B relative to the cosmic etheric frame of reference are $v_{a}$ and $v_{b}$ respectively according to the Lorentz transformation formula, the velocity of particle A relative to particle B should be calculated based on the Galileo transformation when the two particles are far apart, and should be the vector superposition of $v_{a}$ and $v_{b}$. If both $\mathrm{A}$ and $\mathrm{B}$ particles are photons, the velocity of photon A relative to photon B should be $c+c$ (vector superposition) when the two photons are far away from each other. If two photons collide (either believing that photon $A$ enter the etheric layer of photon $B$ or that photon $B$ enter the etheric layer of photon $\mathrm{A}$ ), the Lorentz transform must be used to calculate the collision speed between the two photons that is always $c$ (the constant speed of light).

From the point of view of special relativity, ether does not exist, all inertial systems have equal positions, and the Lorenz transform is suitable for the transformation between any two inertial systems.

So, it can be seen that Lorenz transformation has the above two different understandings and applications. From the point of view of the new etheric view of this paper, the inertial system in special relativity is a mathematical concept that does not exist in the physical world, and in the special relativity the understanding of the physical meaning of Lorenz transformation and the application of Lorenz transformation are wrong.

\subsection{The Absoluteness of Simultaneity}

According to the etheric theory of this paper, two simultaneous events in an etheric system occur simultaneously to any observer. For example, if a light pulse is emitted from the mid-point of a $l_{0}$ long capsule resting in the solar etheric system, the light signal will be received simultaneously by the capsule's head A and tail B to any observer, as shown in Figure 2. Because in this case according to the modified principle of constant speed of light of this paper, the speed of light traveling through the solar ether is constant $c(299,792,458 \mathrm{~m} / \mathrm{s})$, independent of the observer (the result is the same whether observed by observers in the capsule stationary in the solar etheric system or from earth moving relative to the solar etheric system). 


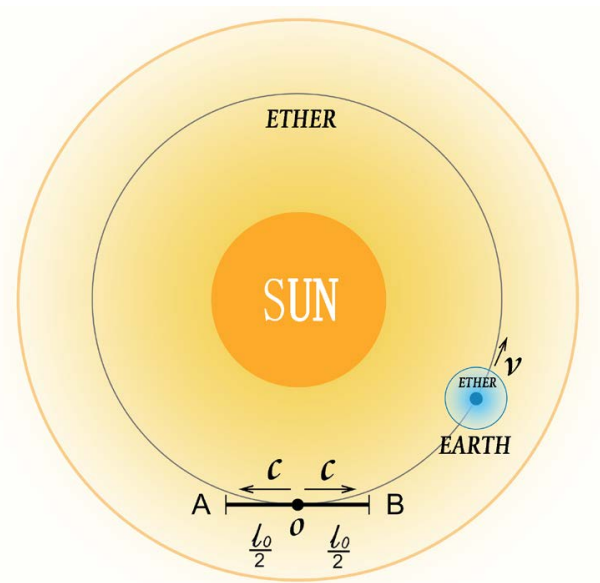

Figure 2. A light pulse is emitted from the mid-point of a $l_{0}$ long capsule resting in the solar etheric system, the light signal will be received simultaneously by the capsule's head A and tail B to any observer.

\section{The Etheric Explanations for the Conclusions of Special Relativity}

According to the new etheric view of this paper, the effect of speed on mass, the effect of speed on time and the effect of speed on length derived from the Lorentz transformation are not the relative observation effects between two different inertial systems, but the absolute real physical effects caused by ether when the object is moving in ether.

\subsection{The Etheric Explanation of the Relation between Mass and Speed}

\subsubsection{Derivation of the Mass-Speed Relationship Formula}

Suppose an object of stationary mass $m_{0}$ is placed stationary in the solar etheric system $S$ and near earth orbit, as shown in Figure 3. When the earth is far away from the object, the object can be thought of as stationary in the solar ether.

When the earth moves to the position of the object and the object is then in the earth's ether, if the object is very close to the earth's surface, the object can be considered to be completely in the earth's ether. At this point, the object moves relative to the ether of the earth at the speed at which the earth revolves around the sun, and the mass of the object is the moving mass $m$, as shown in Figure 4.

We have the Lorentz time transformation formula (4.9):

$$
t^{\prime}=\frac{t-v x / c^{2}}{\sqrt{1-(v / c)^{2}}}
$$

and the Lorentz velocity transformation formula (4.15):

$$
u_{x}=\frac{u_{x}^{\prime}+v}{1+v u_{x}^{\prime} / c^{2}}
$$

Since the object is stationary in the solar etheric system $\mathcal{S}$, we have: 


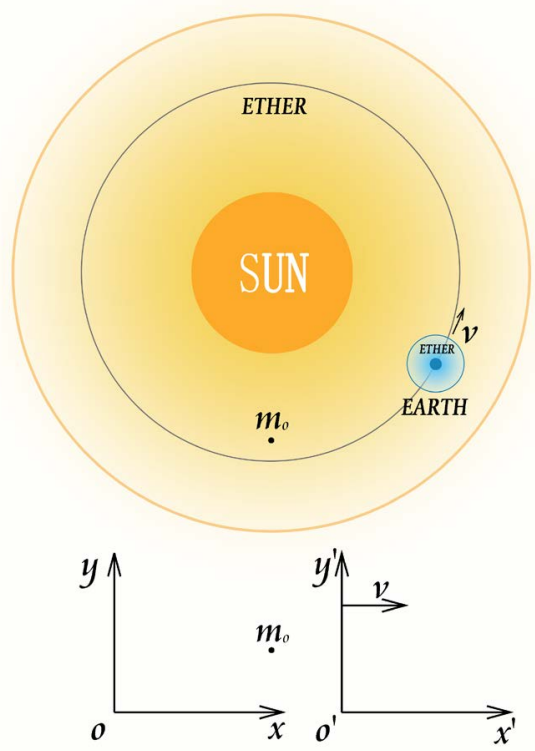

Figure 3. An object of stationary mass $m_{0}$ is placed stationary in the solar etheric system $S$ and near earth orbit.
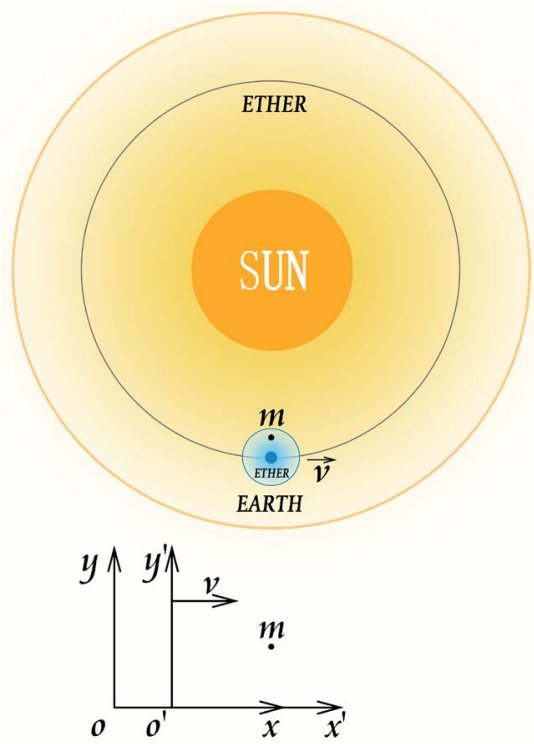

Figure 4. The object moves relative to the ether of the earth at the speed at which the earth revolves around the sun.

$$
u_{x}=0
$$

Bring Equation (5.1) into Equation (4.15), we get:

$$
u_{x}^{\prime}=-v
$$

Since the object is at rest in $S$ system, the mass of the object measured in $S$ system is the object's static mass $m_{0}$. If $F_{x}$ is the external force on the object along the $x$ direction in $S$ system, and $F_{x}^{\prime}$ is the external force on the object along the $x$ ' direction in $S$ 'system, then according to the "modified principle of special relativity" of this paper, we must have: 


$$
F_{x}=F_{x}^{\prime}
$$

Because if an object is not subjected to force and is stationary or moving in a straight line with a uniform speed in the $S$ system, it should also be not subjected to force and be stationary or moving in a straight line with a uniform speed in the $S$ 'system, otherwise Newton's first law can't hold both in the $S$ system and the $S$ 'system. Therefore, according to the modified principle of relativity in this paper that "any law of physics has the same form in all the etheric systems", Equation (5.3) must be true. And because:

$$
\begin{aligned}
& F_{x}=\frac{\mathrm{d} p}{\mathrm{~d} t}=\frac{\mathrm{d}\left(m_{0} u_{x}\right)}{\mathrm{d} t} \\
& F_{x}^{\prime}=\frac{\mathrm{d} p^{\prime}}{\mathrm{d} t^{\prime}}=\frac{\mathrm{d}\left(m u_{x}^{\prime}\right)}{\mathrm{d} t^{\prime}}
\end{aligned}
$$

From Equations (5.3), (5.4) and (5.5) we get:

$$
\mathrm{d}\left(m u_{x}^{\prime}\right)=\frac{m_{0} \mathrm{~d} u_{x} \mathrm{~d} t^{\prime}}{\mathrm{d} t}
$$

Since the object is stationary in $S$ system, $\mathrm{d} x=0$. According to Equation (4.9), we have:

$$
\mathrm{d} t^{\prime}=\frac{\mathrm{d} t}{\sqrt{1-(v / c)^{2}}}
$$

Differential derivation of formula (4.15) we get:

$$
\mathrm{d} u_{x}=\mathrm{d}\left(\frac{u_{x}^{\prime}+v}{1+v u_{x}^{\prime} / c^{2}}\right)=\frac{\left(1-v^{2} / c^{2}\right) \mathrm{d} u_{x}^{\prime}}{\left(1+v u_{x}^{\prime} / c^{2}\right)^{2}}
$$

Substitute Equation (5.1), (5.7) and (5.8) into Equation (5.6), and notice that $u_{x}^{\prime}=-v$, we get:

$$
\mathrm{d}\left(m u_{x}^{\prime}\right)=m_{0} \frac{\mathrm{d} u_{x}^{\prime}}{\sqrt{\left(1-\frac{v^{2}}{c^{2}}\right)^{3}}}
$$

and:

$$
\mathrm{d}(m v)=m_{0} \frac{\mathrm{d} v}{\sqrt{\left(1-\frac{v^{2}}{c^{2}}\right)^{3}}}
$$

Integrate both sides of Equation (5.10), we get:

$$
m v=\frac{m_{0} v}{\sqrt{1-\frac{v^{2}}{c^{2}}}}
$$

that is:

$$
m=\frac{m_{0}}{\sqrt{1-\frac{v^{2}}{c^{2}}}}
$$




\subsubsection{The Physical Mechanism of the Mass-Speed Relationship}

In the formula (5.12), $v$ is the speed of the object relative to the ether when the object is moving in the ether, $m_{0}$ is the inertia mass of the object at rest relative to the ether, and $m$ is the inertial mass of the object moving relative to the ether with speed $v$. So, when the speed of an object moving relative to the etheric system is fixed, the inertial mass of the object is the same no matter what reference frame is adopted for observation. For example, in the solar etheric system, the inertial mass (kinetic energy part) of the Earth is determined by the speed of the Earth relative to the solar etheric system (the etheric layer dragged together by the sun), and the results are the same whether the observer observes on Earth, on the moon or on Mars.

In the galactic etheric system, the inertial mass (kinetic energy part) of the Earth is determined by the speed of the Earth relative to the galactic etheric system, and the results are the same whether the observer observes on Earth, on the moon or on Mars.

In the etheric system of the universe, the inertial mass (kinetic energy part) of the Earth is determined by the speed of the Earth relative to the etheric system of the universe, and the results are the same whether the observer observes on Earth, on the moon or on Mars.

Because an object that moves in ether can contact more etheric particles (assuming that ether is made up of very tiny particles) in a unit time than an object that is at rest in the ether. Moreover, the faster the object moves, the more etheric particles are contacted per unit time, the more etheric particles are attracted and the higher the density of etheric particles is in the object. This is why the inertial mass of an object increases as it moves in the ether, and the faster the speed, the larger the inertial mass. It is also because the increase of the etheric density of space can lead to the slowness of time, so formula (5.12) is formed.

From the above discussions, we can get the conclusion that the higher the etheric density of the space, the greater the mass of the object, and that with the expansion of the universe, the etheric (energy) density of space is decreasing, so the mass of all objects and particles is decreasing.

\subsection{The Etheric Explanation of the Relation between Time and Speed}

\subsubsection{Derivation of the Time-Speed Relationship Formula}

Suppose an object is placed stationary near earth orbit in the solar etheric system $S$, as shown in Figure 5. When the earth is far away from the object, the object can be thought of as stationary in the solar ether. Assuming that in the $S$ system there is a static clock and that two events (such as the birth and death of a bacterium) occur on the object one after another, and that the two events are recorded by the clock at $t_{1}$ and $t_{2}$ respectively, the time interval between the two events in the $S$ system is as follows:

$$
\Delta t=t_{2}-t_{1}=t_{0}
$$

In the above formula, $t_{0}$ is called the proper time. 


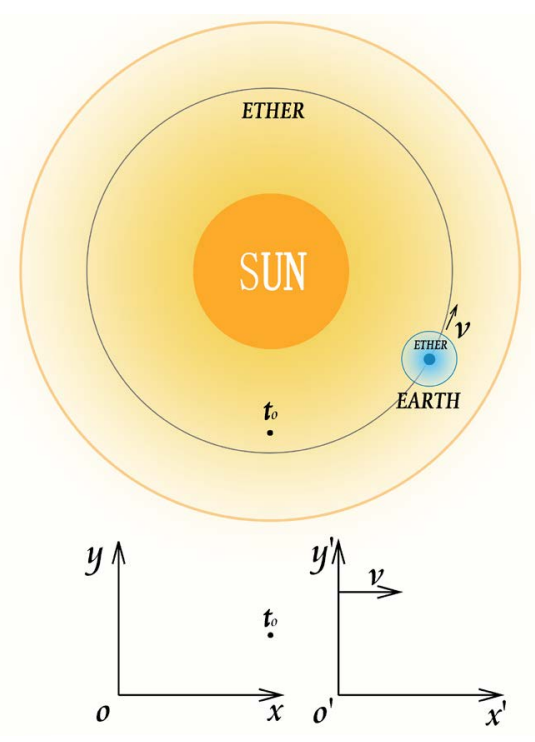

Figure 5. An object is placed stationary near earth orbit in the solar etheric system $S$, and two events occur on the object one after another. The time interval between the two events is $t_{0}$.

When the earth moves to the position of the object and the object is in the earth's ether, if the object is very close to the earth's surface, the object can be considered to be completely in the earth's ether. At this point, the object moves relative to the ether of the earth at the speed at which the earth revolves around the sun, as shown in Figure 6. Assuming that in the $S$, system there is also a static clock, and that the same two events (such as the birth and death of the same bacterium) occur on the object one after another and are recorded by this clock at $t_{1}^{\prime}$ and $t_{2}^{\prime}$ respectively, the time interval between the two events in the $S$ 'system is as follows:

$$
\Delta t^{\prime}=t_{2}^{\prime}-t_{1}^{\prime}=t
$$

From Lorentz transformation (4.9) we have:

$$
\begin{aligned}
& t_{1}^{\prime}=\frac{t_{1}-v x / c^{2}}{\sqrt{1-(v / c)^{2}}} \\
& t_{2}^{\prime}=\frac{t_{2}-v x / c^{2}}{\sqrt{1-(v / c)^{2}}}
\end{aligned}
$$

From Equations (5.13), (5.14), (5.15) and (5.16), we can get:

$$
t=\frac{t_{0}}{\sqrt{1-\frac{v^{2}}{c^{2}}}}
$$

\subsubsection{The Physical Mechanism of the Time-Speed Relationship}

In formula (5.17) $v$ is the speed of the object moving relative to the ether, and $t_{0}$ is the time interval between the two events when the object is at rest in ether, $t$ is time interval between the two events when the object is moving relative to the ether with speed $v$. 


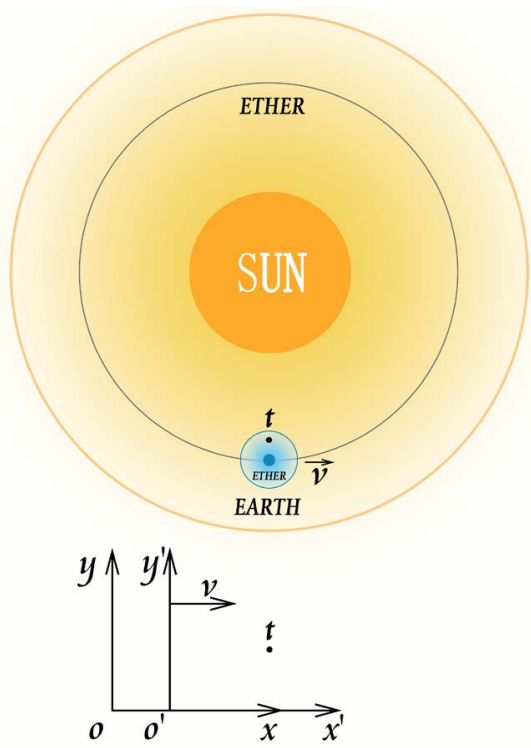

Figure 6. The object moves relative to the ether of the earth at the speed at which the earth revolves around the sun, and the same two events occur on the object. The time interval between the two events is $t$.

Because the etheric density in the space in an object will increase as the object moves in the ether, and the faster the speed, the greater the etheric density of the space in the object. The increase in the etheric density of the space in the object will slow down the time on the object. So, the speed effect of time is not the relative observation effect produced by the relative motion of the two inertial systems, but a real and absolute physical effect given by ether when the object moves in the ether (independent of the observer).

It can be seen from the formula (5.17) that with the decelerating expansion of the universe, the speed of all the objects relative to the universe etheric system decrease, or the inertial mass of all the objects decrease constantly, and the time on all the objects will continue to accelerate.

\subsubsection{The Exploration for the Essence of Time}

We know that the stronger the gravitational field is, the higher the density of the ether in the space of the gravitational field, and now we also know that the faster the object moves in ether, the higher the density of the ether in the space inside the object. So from the facts that time slows down with the gravitational field's increase, and slows down with the increase of the speed of the object relative to the ether, we can get the following conclusion: the time at a point in cosmic space during cosmic expansion (during which the ether flows from particles to cosmic space) slows down (speeds up) as the density of the ether at that point increases (decreases). Or, the product of the time and the density of ether at any point of the space is the same:

$$
\rho t=b
$$

In the formula, $\rho$ is the etheric (energy) density of a point in the space, $t$ is 
the time at the same point, and $b$ is a constant (the unit is Joule second/cubic meter). By formula (5.18), it can be seen that there is no meaning for the time in the case that the ether density is zero because the speed of time flow is infinite.

During cosmic contraction (during which the ether flows from space to particles), time at a point in space speeds up (slows down) as the density of the ether (energy) at that point increases (decreases). Or, the product of the time and the reciprocal of density of ether at any point of the space is the same.

It can be seen that time is the flow rate of ether from the object to the space during cosmic expansion or the flow rate of ether from the space to the object during cosmic contraction.

From the above discussions, we can also get the conclusions that the higher the etheric density of the space, the slower the time on the object, and that with the expansion of the universe, the etheric (energy) density of space is decreasing, the time is accelerating.

\subsection{The Etheric Explanation of the Relation between Length and Speed}

\subsubsection{Derivation of the Length-Speed Relationship Formula}

Let $S$ and $S^{\prime}$ be two etheric systems, one large and one small, the small etheric system $S$ '(for example, the Earth etheric system) is in the large etheric system $S$ (for example, the solar etheric system) and is moving in a straight line with uniform speed relative to $S$. Suppose a thin rod is placed stationary along the direction of earth's motion in the position near the earth's orbit in the solar etheric system $S$, as shown in Figure 7. When the earth is far away from the rod, the rod can be regarded as stationary in the solar ether. At this time, for any observer, the coordinates of both ends of the rod, $x_{1}$ and $x_{2}$, are measured simultaneously (according to section 4.4, simultaneity is absolute), and the length of the measured rod is:

$$
l_{0}=x_{2}-x_{1}
$$

In the formula, $l_{0}$ is the prober length.

When the earth moves to the position of the rod and the rod is in the earth's ether, if the rod is very close to the earth's surface, the rod can be considered to be completely in the earth's ether. At this point, the rod moves relative to the ether of the earth at the speed at which the earth revolves around the sun, as shown in Figure 8. At this time, for any observer, the coordinates of both ends of the rod, $x_{1}^{\prime}$ and $x_{2}^{\prime}$, are measured simultaneously (according to section 4.4, simultaneity is absolute), and the length of the measured rod is:

$$
l=x_{2}^{\prime}-x_{1}^{\prime}
$$

From Lorentz transformation (4.10) we have:

$$
x_{1}=\frac{x_{1}^{\prime}+v t_{1}^{\prime}}{\sqrt{1-(v / c)^{2}}}
$$




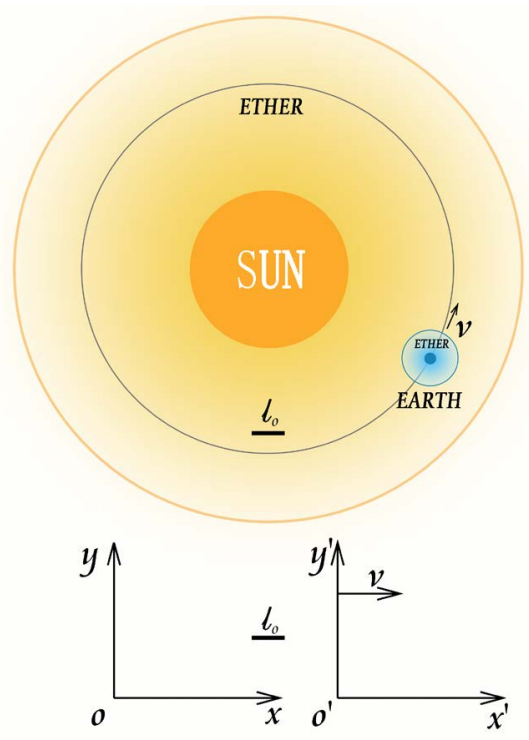

Figure 7. A thin rod is placed stationary along the direction of earth's motion in the position near the earth's orbit in the solar etheric system $S$.
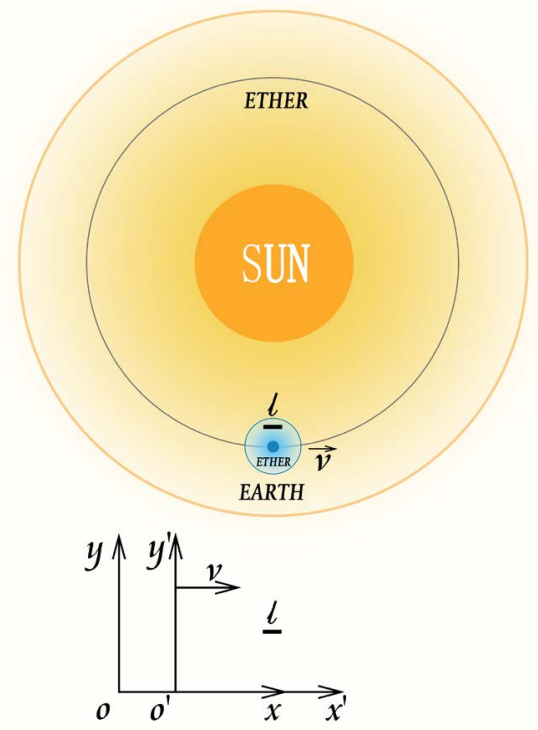

Figure 8. The rod moves relative to the ether of the earth at the speed at which the earth revolves around the sun.

$$
x_{2}=\frac{x_{2}^{\prime}+v t_{2}^{\prime}}{\sqrt{1-(v / c)^{2}}}
$$

In the formula $t_{1}^{\prime}=t_{2}^{\prime}$. Subtract the above two equations we get:

$$
x_{2}-x_{1}=\frac{x_{2}^{\prime}-x_{1}^{\prime}}{\sqrt{1-(v / c)^{2}}}
$$

That is:

$$
l=l_{0} \sqrt{1-\frac{v^{2}}{c^{2}}}
$$


where $V$ is the speed of the rod relative to the ether, and $l_{0}$ is the length when the rod is at rest in ether, $l$ is the length when the rod is moving relative to the ether with speed $v$.

\subsubsection{The Physical Mechanism of the Length-Speed Relationship}

The speed effect of length is not the relative observation effect produced by the relative motion of the two inertial systems, but a real and absolute physical effect given by ether when the object moves in the ether (it has nothing to do with the observer).

The size of an object is determined by the average distance between the nucleuses of the object, and the average distance between the nucleuses is determined by the average size of the atoms composed of the object. The size of the atom is determined by the inertial mass of the electron and the coulomb force between the nucleus and the electron.

From the coulomb force formula between the electron and the nucleus [8]:

$$
F_{e}=\frac{1}{4 \pi \varepsilon_{0}} \frac{n e^{2}}{r_{a}^{2}}
$$

where $F_{e}$ is the coulomb force between the electron and the nucleus, $e$ is the electric charge of the electron, $n$ is the number of proton of the nucleus, $r_{a}$ is the distance between the electron and nucleus, $\varepsilon_{0}$ is the dielectric constant in vacuum. The formula of the inertial centrifugal force of the electron [9]:

$$
F_{I}=\frac{m v_{e}^{2}}{r_{a}}
$$

where $F_{I}$ is the inertial centrifugal force of the electron orbiting the nucleus, and $m$ is the inertial mass of the electron; $v_{e}$ is the speed of the electron orbiting the nucleus. The formula of angular momentum conservation that the electron orbits the nucleus [10]

$$
m v_{e} r_{a}=b=n h, n=1,2,3, \cdots
$$

where $b$ is invariant, $h$ is planck constant. And the following equation:

$$
F_{e}=F_{I}
$$

We can get the radius of the electron orbiting the nucleus:

$$
r_{a}=\frac{4 \pi \varepsilon_{0} b^{2}}{n e^{2} m}
$$

and the speed of electron orbiting the nucleus:

$$
v_{e}=\frac{n e^{2}}{4 \pi \varepsilon_{0} b}
$$

From (5.12) and (5.29) we can get:

$$
r_{a}=\frac{4 \pi \varepsilon_{0} b^{2}}{n e^{2} m_{0}} \sqrt{1-\frac{v^{2}}{c^{2}}}=r_{a 0} \sqrt{1-\frac{v^{2}}{c^{2}}}
$$

In the formula $r_{a 0}$ is the radius of the atoms that makes up the object as the 
object is at rest relative to the ether, and $\mathrm{v}$ is the speed at which the object moves relative to the ether.

From formula (5.31) we can see that the faster the object moves relative to the ether, the greater the contraction of the atoms of the object, and the greater the contraction of the object. It can also be said that the faster the object moves relative to the ether, the higher the etheric density of the inner space of the object (because the kinetic energy of the object is greater), and the higher the etheric density of the inner space of the object, the more the object contracts.

By (5.31) we see that with the decelerating expansion of the universe, or as the object slows down along the direction of the universe expansion, the speed of the object relative to the cosmic etheric system decreases, the etheric density of the object decreases, the object expands.

From what has been discussed above, we can get the following conclusions: The higher the etheric density of space, the greater the contraction of the objects in space.

Corollary 1: Because the etheric (energy) density of space decreases with the decelerating expansion of the universe, all objects (stars, planets, molecules, atoms, etc.) in the universe expand as the universe expansion slows.

Corollary 2: Because the stronger the electric or magnetic field, the higher the density of the ether (energy) in space, the stronger the electric or magnetic field, the more shrinking the object in the electric or magnetic field.

\subsubsection{The Exploration for the Essence of Space}

As can be seen from section 5.2.3, time is meaningless in a vacuum. In addition, according to the discussions in the previous sections of this paper, all the laws of physics can hold only in the etheric system, so the vacuum is meaningless to physics. It follows that the space of physics is the space filled with ether, and that the essence of space is ether.

\subsection{The Etheric Explanation of the Relation between Mass and Energy}

According to the definition of momentum and the mass velocity relationship (5.12), we have:

$$
p=\frac{m_{0} v}{\sqrt{1-v^{2} / c^{2}}}
$$

Suppose a particle, under the action of a variable force, starts at rest and moves along the $\mathrm{x}$-axis in one dimension. When the velocity of the particle is $\mathrm{V}$, its kinetic energy is:

$$
E_{k}=\int F_{x} \mathrm{~d} x=\int \frac{\mathrm{d} p}{\mathrm{~d} t} \mathrm{~d} x=\int v \mathrm{~d} p=p v-\int_{0}^{v} p \mathrm{~d} v
$$

From Equations (5.32) and (5.33) we get

$$
E_{k}=m c^{2}-m_{0} c^{2}
$$




$$
m c^{2}=E_{k}+m_{0} c^{2}=E
$$

That is:

$$
E=m c^{2}=\frac{m_{0} c^{2}}{\sqrt{1-v^{2} / c^{2}}}
$$

In formula (5.36), $V$ is the speed of the object relative to the etheric system, $m c^{2}$ is the energy (ether content) of the object when the object moves relative to the etheric system with speed $v, m_{0} c^{2}$ is the energy (ether content) of the object when the object is stationary relative to the etheric system. From formula (5.36) we see that the faster the object moves in the ether, the more the ether collected by the object (the higher the ether density of the object), and the greater the kinetic energy of the object.

\section{Relevant Experimental Verifications}

So far, all the experiments that have verified the correctness of special relativity (such as the experiments of the constancy of light velocity, the experiments of the relationship between time and speed, the experiments of the relationship between length and speed, the experiments of the relationship between mass and speed, the experiments of mass and energy formula or the explosion of atomic bombs, etc.) can also be explained by the theory of this paper. Therefore, these experiments have now proved that both theories are correct. Which of the two is the right one must be identified by additional experiments. The following are several additional experiments designed:

\subsection{Experiment 1: Verify That the Kinetic Energy of a Particle Is Only Related to the Speed of the Particle Relative to the Ether, Independent of the Observer's Motion}

Do the following particle collision experiments on cyclotron:

Collision experiment 1: Let two of the same particle A and $\mathrm{B}$ to move with a speed of $0.5 C$ and $-0.5 c$ relative to the laboratory frame of reference respectively and collide. Measure the collision energy produced by the collision.

Collision experiment 2: Two of the same particle A and B, let B be stationary relative to the laboratory frame of reference and A move with a speed of (4/5) $C$ relative to the laboratory frame of reference respectively, and let the two particles collide. Measure the collision energy produced by the collision. Comparing with the above two experiments, and it will be verified that the collision energy produced by two collision events is different. But if take B particle as the reference frame, then the collision energy of two collision events calculated according to the theory of Special Relativity is the same.

In collision experiment 1 , the collision energy calculated with particle A or B

as the reference frame is $\frac{5}{3} m_{0} c^{2}$. It will be proved that the calculated result is not consistent with the experimental result. 
In collision experiment 2 , the collision energy calculated with the laboratory reference frame is $\frac{5}{3} m_{0} c^{2}$. It will be proved that the calculated result is consistent with the experimental result.

\subsection{Experiment 2: Verify That Magnetic Field Is Produced by Electric Charge's Motion Relative to Ether, and Is Independent of the Observer}

According to the theory of this paper, the Earth is dragging the ether to move together (the ether near the surface of the Earth is stationary relative to the surface of the Earth), and that the production of the magnetic field by the motion of the electric charge to ether is an absolutely real physical effect, and has nothing to do with the observer. The motion that the electric charged pole is stationary relative to the ether but the compass is moving relative to the pole is different from motion that the compass is stationary relative to the ether and the electric charged rod is moving relative to the compass, and will produce different physical effect. The former does not generate a magnetic field in space (the compass does not rotate), while the latter produces a magnetic field in space (the compass rotates).

Expected experimental results:

When the charged bar is stationary relative to the laboratory (etheric system of the Earth), no matter how the compass moves relative to the charged metal bar, the compass will not rotate.

When the charged bar moves relative to the laboratory (etheric system of the Earth) the compass rotates regardless of whether it is stationary or moving to the charged bar.

We can consider to put an electric charged rod and a compass on the table of a high-speed train (let charged rod and a compass close to each other), let the charged rod point to the direction of the high-speed train movement. When the train is moving and the compass is stationary relative to the charged rod, turn the compass to see if it will turn back or not. When the train is stationary and the compass is stationary relative to the charged rod, turn the compass to see if it will turn back or not.

Expected experimental results: When the high-speed train moves, there is magnetic field generated; when the high-speed train stops moving, there is no magnetic field generated.

This experiment will prove that the electric charge generates magnetic field when it moves relative to ether, and that the production of the magnetic field by the motion of the electric charge to "ether" is an absolutely real physical effect, and has nothing to do with the observer

\subsection{Experiment 3: Redoing the Michelson-Morley Experiment on the Space Station}

So far all measurements of the "etheric wind", such as the Michelson-Morley 
experiment [3], have been carried out on the ground. According to the theory of this paper, the Earth drags ether to move together, and ether is stationary to the Earth's surface. Therefore, all the measurements of "etheric wind" done on the Earth's surface can't detect the "etheric wind". That the space station does not fall off is because of the centrifugal force created by the space station's motion relative to the ether of the Earth. So, there should be an "etheric wind" in the space station. In addition, as the etheric layers dragged by the wall of the space station and the experimental station are very thin, the light can be avoided to pass through these etheric layers during the experiment.

Prediction: The etheric wind will be detected if the Michelson-Morley experiment is done on the space station.

This experiment will prove that ether exists.

\subsection{Experiment 4: Simulation of the Generation and Reversal of the Earth's Magnetic Field, Thus Proving That the Magnetic Field Is Generated by the Relative Motion of the Electric Charge to the Ether}

Put a large spherical shell cover a small spherical shell of similar radius concentrically and charge the two spherical shells with equal but opposite static charges. Let the outer spherical shell rest, the inner spherical shell rotates, from the axis of rotation a magnetic field will emit out. Remove the outer shell and rotate the inner shell with the same speed, you will see that the magnetic field is much weaker. The experimental results show that the electrostatic field generated by the charged inner spherical shell gathers the ether, and the charged outer spherical shell rotates in the ether of the inner spherical shell, thus producing a magnetic field. The experiment will prove that the magnetic field is produced by the relative motion of the electric charge to the ether.

Let the outer spherical shell rotate and Let the rotating axis of the inner spherical shell periodically change 180 degrees relative to the rotating axis of the outer spherical shell in a short time. A reversal of the magnetic pole relative to the outer spherical shell occurs at each turn of 180 degrees. This experiment will reveal the physical mechanism of the generation and reversal of the Earth's magnetic field [11]

\subsection{Experiment 5: Simulate the Formation of the Natural Iron Meteorites and Shows That the Atom Becomes Smaller with the Increase of the Etheric Density of the Space}

Cool and crystallize the molten iron in strong magnetic field. Place this crystallized iron in an easily oxidized environment for antioxidant experiments.

Prediction: such iron is just as iron meteorites that is not easy to rust. Because the density of ether in strong magnetic field is very high, and the atom becomes smaller with the increase of etheric density in space, the density of iron condensed in strong magnetic field is higher than that of normal iron, which is not easy to react with oxygen molecule. 


\subsection{Experiment 6: Aberrations Contrast Experiment}

Install an arm on the wall of a tall building at the high place, and Install a light source and suspend a heavy weight object on the arm respectively at a certain distance from wall of the building (such as $1.5 \mathrm{~m}$ and $1 \mathrm{~m}$ ). Let the weight object be close to the ground. Install an observing instrument on the ground under the light source vertically to measure the aberration. If there is an aberration, the aberration angle (the angle between the observer and the vertical line of the suspended heavy object) produced by the position of the light source observed in the observing instrument is the same as that of the star. If there is no aberration, there is no aberration angle for the light source position seen by the observer (the observer is parallel to the vertical line of the weight suspended).

According to special relativity, since the constant velocity of light in vacuum is independent of the motion of the light source, the observation of the light source on top of a building and the observation of the stars should have the same aberration effect because both are caused by the movement of the Earth around the sun and the rotation of the Earth.

According to the theory of this paper, since the light source on the building and the observer are both in the Earth's etheric system, the photons emitted by the light source remain constant speed relative the Earth etheric system, while the observer is stationary relative to the Earth etheric system, so there should be no aberration effect. Because the light from a star must first enter the solar etheric system before it can enter the Earth's etheric system, and the Earth's etheric system has a relative motion with the sun's etheric system (caused by the movement of the Earth around the sun and the rotation of the Earth), there will be an aberration effect of the star for the observers on Earth.

The aberration angle $\phi$ can be calculated by the Lorentz velocity transformation (4.14) between the solar etheric system and the Earth etheric system:

$$
\operatorname{tg} \phi=\frac{u_{y}^{\prime}}{u_{x}^{\prime}}=\frac{u_{y}}{\gamma\left(u_{x}-v\right)}=\frac{\sin \theta}{\gamma(\cos \theta-v / c)}
$$

In the above formula, $u_{y}^{\prime}$ and $u_{x}^{\prime}$ are the vertical and horizontal velocity components of the photon in the Earth etheric system respectively, $u_{y}$ and $u_{x}$ are the vertical and horizontal velocity components of the photon in the solar etheric system respectively, $v$ is the speed of the Earth's etheric system relative to the sun's etheric system, $\mathrm{c}$ is the speed of light in vacuum.

$$
\gamma=1 / \sqrt{1-(v / c)^{2}}
$$

In conclusion: for the observers on Earth, according to the theory of this paper, stars have aberration but the light sources on Earth have no aberration; according to the theory of special relativity, there are aberrations for both the stars and the light sources on Earth. 


\section{Conclusions}

1) The cosmic space is filled with ether (energy), which forms the cosmic absolute static frame of reference and is named the cosmic etheric system.

2) Each particle or object carries an etheric layer (sphere of influence) that surrounds and moves with it. The etheric layer forms an absolute frame of reference and is named the particle or object etheric system.

3) Among all the etheric systems, only the cosmic etheric system is an isolated system, and all the conservation laws (such as energy conservation, momentum conservation, and angular momentum conservation, etc.) are strictly valid only in the cosmic etheric system.

4) The propagation speed of light in all etheric systems remains constant $C$ $(299,792,458 \mathrm{~m} / \mathrm{s})$, which has nothing to do with the motion of the light source and the motion of the etheric system.

5) The form of any law of physics is the same in all kinds of etheric systems.

6) The Lorentz transformation is only suitable for two related etheric systems (such as the Earth etheric system and the solar etheric system).

7) The effect of speed on mass, the effect of speed on time and the effect of speed on length derived from the Lorentz transformations are not the relative observation effects between two different inertial systems, but the absolute real physical effects caused by ether when the object is moving in ether.

8) The higher the etheric density of the space, the greater the mass of the object, the more the object contracts, and the slower the time on the object.

\section{Conflicts of Interest}

The author declares no conflicts of interest regarding the publication of this paper.

\section{References}

[1] Tan, S.H. (1987) The Study of Dialectics of Nature, No. 3, 7-17.

[2] Michelson, A.A., Pease, F.G. and Pearson, F. (1929) Journal of the Optical Society of America, 18, 181-181.

[3] Einstein, A. (1905) Annalen der Physik, 17, 891-921.

[4] Halliday, D., Resnick, R. and Walker, J. (2001) Fundamental of Physics. Chapter 38, Relativity, Sixth Edition, Wiley, New York, 932-933.

[5] Halliday, D., Resnick, R. and Walker, J. (2001) Fundamental of Physics. Sixth Edition, Wiley, New York, 960.

[6] Halliday, D., Resnick, R. and Walker, J. (2001) Fundamental of Physics. Sixth Edition, Wiley, New York, 762.

[7] Cutnell, J.D. and Johnson, K.W. (2001) Physics. Fifth Edition, Wiley, New York, 804.

[8] Halliday, D., Resnick, R. and Walker, J. (2001) Fundamental of Physics. Sixth Edition, Wiley, New York, 524.

[9] Cutnell, J.D. and Johnson, K.W. (2001) Physics. Fifth Edition, Wiley, New York, 134. 
[10] Cutnell, J.D. and Johnson, K.W. (2001) Physics. Fifth Edition, Wiley, New York, 917-922.

[11] Jacobs, J. (1995) Geophysical Journal International, 121, 574-575. 